\title{
Clinical significance of high on-treatment platelet reactivity in patients with prolonged clopidogrel therapy
}

\author{
Sehun Kim ${ }^{1}$ Donghoon $\mathrm{Han}^{2}$, Jae Hyuk Choi ${ }^{3}$ Eun-Joo Park², Dong Geum Shin², Min-Kyung Kang ${ }^{2}$, \\ Seonghoon $\mathrm{Choi}^{2}$, Namho Lee ${ }^{2}$, and Jung Rae $\mathrm{Cho}^{2}$
}

${ }^{1}$ Division of Cardiology, Department of Internal Medicine, Seongnam Citizens Medical Center, Seongnam; ${ }^{2}$ Division of Cardiology, Department of Internal Medicine, Hallym University Kangnam Sacred Heart Hospital, Seoul; ${ }^{3}$ Division of Cardiology, Department of Internal Medicine, Hallym University Dongtan Sacred Heart Hospital, Hwaseong, Korea

Received: March 4, 2020

Revised : June 23, 2020

Accepted: August 3, 2020

\section{Correspondence to}

Jung Rae Cho, M.D.

Division of Cardiology,

Department of Internal

Medicine, Hallym University

Kangnam Sacred Heart Hospital,

1 Singil-ro, Yeongdeungpo-gu,

Seoul o7441, Korea

Tel: $+82-2-829-5393$

Fax: $+82-2-849-4460$

E-mail:jrjoe@hallym.or.kr https://orcid.org/0000-00029803-6612
Background/Aims: Prolonged dual antiplatelet therapy (DAPT) with aspirin and clopidogrel beyond 1 year has been shown to reduce ischemic events at the expense of increased bleeding. However, limited data are available on the clinical significance of platelet reactivity (PR) at 1 year.

Methods: We retrospectively identified 331 patients who underwent percutaneous coronary intervention (PCI) and assessed the on-clopidogrel PR using VerifyNow $\mathrm{P}_{2} \mathrm{Y}_{12}$ assay at 1 year in a single center. Two hundred eleven patients were on DAPT for $>1$ year. The relationship between high on-treatment platelet reactivity (HPR) at 1 year and clinical outcomes beyond 1 year, as well as the longitudinal change in PR was analyzed.

Results: At 1 year, 135 (64\%) patients showed HPR and 76 (36\%) did not. There was a significant increase in ischemic endpoint events, including cardiovascular death, non-fatal myocardial infarction, and stroke/transient ischemic attack in patients with compared to without HPR at 1 year (hazard ratio [HR], 2.68; 95\% confidence interval [CI], 1.06 to 6.77; $p=0.036$ ). However, the incidence of any Bleeding Academic Research Consortium bleeding was significantly lower in the HPR group (HR, 0.11; 95\% CI, 0.02 to $0.65 ; p=0.015$ ). In the longitudinal analysis, PR significantly decreased from post-load to 1 year after index PCI in the non-HPR group. Conversely, the HPR group showed high PR from baseline through 1 year.

Conclusions: HPR at 1 year may be a useful surrogate for predicting ischemic and bleeding events in patients on prolonged DAPT. Patients with and without HPR at 1 year showed different patterns of longitudinal change in PR.

Keywords: Dual anti-platelet therapy; Percutaneous coronary intervention; Platelet function tests; Retrospective study

\section{INTRODUCTION}

Coronary artery disease (CAD) continues to be a leading cause of mortality and morbidity worldwide [1]. Percutaneous coronary intervention $(\mathrm{PCI})$ with a drug-eluting stent (DES) and pharmacological therapy with aspirin and $\mathrm{P}_{2} \mathrm{Y}_{12}$ inhibitors have improved clinical outcomes and have become the cornerstone therapies. However, stent thrombosis remains a major concern in patients with DES owing to its high morbidity and mortality $[2,3]$.

Platelets play a key role in the pathophysiology of both stent thrombosis and intracoronary thrombosis arising from ruptured atherosclerotic plaques. Thus, antiplatelet therapy, particularly dual antiplatelet therapy (DAPT), 
is recommended for at least 6 months in patients with stable ischemic heart disease or for 12 months in patients with acute coronary syndrome (ACS) after DES implantation (class I strength of recommendation), and for a prolonged duration in patients without treatment complications (class IIb strength of recommendation) [4].

Aspirin and clopidogrel are the most common combination drugs in patients undergoing PCI, although variability in the response to clopidogrel has been reported [5]. The clinical implications of variability in clopidogrel response have also been demonstrated, including stent thrombosis, recurrent myocardial infarction (MI), and death [6]. Identification of patients with high platelet reactivity (PR) despite clopidogrel treatment is therefore crucial for ensuring safety and improving clinical outcomes.

High on-treatment platelet reactivity (HPR) identified after a loading dose of clopidogrel has been reported to be associated with poor prognosis within 1 year $[7,8]$. However, there is a paucity of data on the clinical implication of HPR at 1 year after index PCI on long-term cardiovascular outcomes. Furthermore, few studies have evaluated the longitudinal changes in PR from the initial to the mid-term periods. Moreover, the optimal timing for platelet function measurement, with respect to fluctuations as seen in the early period of ACS, is still debated $[8,9]$.

This study aimed to provide information on the above-mentioned issues by measuring PR at 1 year, focusing on the HPR status at 1 year compared with the post-load value as well as the clinical outcome beyond 1 year. We sought to investigate (1) the HPR at 1 year and the incidence of cardiovascular death, non-fatal MI, and stroke/transient ischemic attack (TIA) events; (2) the HPR at 1 year and the incidence of bleeding events according to the Bleeding Academic Research Consortium (BARC) classification during the follow-up period beyond 1 year; and (3) the longitudinal change in PR from baseline, to post-load, and to 1 year after index PCI in patients who underwent DES-based PCI.

\section{METHODS}

\section{Study participants and design}

This retrospective study aimed to investigate the influ- ence of HPR status on long-term clinical outcomes beyond 1 year as well as the longitudinal changes in PR. The study was approved by the Institutional Review Board (IRB) of Hallym University Kangnam Sacred Heart Hospital, South Korea (IRB No. 2016-05-55). The study participants were identified and included from Kangnam Sacred Heart Hospital between September 2009 and July 2013, with all patient records analyzed up to June 2016. Written informed consent was not obtained from each subject since this study focused on the observation of PR among the study subjects. The platelet function test using the VerifyNow P2Y12 assay (Accriva Diagnostics, San Diego, CA, USA) was performed as a routine clinical practice. All patients who underwent PCI with DES for either ACS or stable CAD, and on continued DAPT with aspirin and clopidogrel at 1 year after the index PCI were eligible and included in the study. The exclusion criteria were as follows: (1) use of periprocedural glycoprotein IIb/IIIa inhibitors; (2) failure to attend follow-up; (3) uncertain CAD diagnosis; (4) not severe enough CAD to warrant stent placement; and (5) no platelet function test at 1 year. The study protocol conformed to the ethical guidelines of the 1975 Declaration of Helsinki.

\section{Study protocol}

The treatment regimen in all patients was an initial loading dose of $300 \mathrm{mg}$ aspirin and $600 \mathrm{mg}$ clopidogrel. All patients continued their antiplatelet regimen with maintenance doses of $100 \mathrm{mg}$ aspirin and $75 \mathrm{mg}$ clopidogrel once daily for up to 1 year. The decision to continue aspirin alone as a single antiplatelet therapy or to continue DAPT (prolonged DAPT) was made at 1 year after PCI, according to the clinician's discretion. Any cases of cardiovascular and bleeding events from 1 year after the index PCI up to the point of data collection were collected and analyzed. The primary outcome was ischemic endpoint events, which included cardiovascular death, non-fatal MI, and stroke/TIA, and the secondary outcome was any-BARC bleeding $[3,10,11]$.

\section{VerifyNow $\mathrm{P}_{2} \mathrm{Y}_{12}$ assay}

PR was measured using a turbidimetric-based optical detection system (VerifyNow $\mathrm{P}_{2} \mathrm{Y}_{12}$ ), which has been described elsewhere [12]. The degree of PR in conjunction with clopidogrel use was reported as a $\mathrm{P}_{2} \mathrm{Y}_{12}$ reaction unit (PRU), which was measured at baseline, at 24 hours 
after the loading dose, and at 1 year. HPR was defined as a PRU > 208 [13,14]. This method of platelet function analysis is an ideal point-of-care measurement and a sensitive and reliable measure of platelet inhibition with clopidogrel. It has also been shown to discriminate patients with endpoint events within 1 year of follow-up [15].

\section{Statistical analysis}

Baseline characteristics, medications, and diagnoses were also collected and analyzed. GPower 3.1 was used to calculate the power of this study. For a power of $80 \%$ and an alpha risk of $5 \%$, we calculated that 64 patients per group (128 in total) would be required to detect a significant difference while setting the statistical results as means. Statistical analysis was performed using SPSS version 24.0 software (IBM Co., Armonk, NY, USA). Continuous variables are presented as mean \pm standard deviation and were analyzed using Student's $t$ test and analysis of variance (ANOVA). Categorical variables were analyzed using the chi-square test, and when the expected frequencies of observations were $<5$, Fisher's exact test was used. Longitudinal change in PR was analyzed using repeated measures ANOVA. Survival analysis of ischemic and bleeding endpoints was performed using Kaplan-Meier estimates, and the log-rank test was used to compare survival curves between the two groups. A multivariable-adjusted Cox proportional regression hazard model was used to investigate the independent effect of the HPR at 1 year on the clinical outcomes. The Cox model included age $>65$ years, sex, prior MI, stroke/TIA, hypertension (HTN), diabetes mellitus, atrial fibrillation, dyslipidemia, chronic kidney disease, and smoking status (non-smoker vs. current and ex-smoker). The adjusted hazard ratio (HR) with 95\% confidence interval (CI) was calculated. A $p<0.05$ was used to indicate statistical significance.

\section{RESULTS}

\section{Study flow}

A total of 344 patients were identified for this study. Valid PR measurements were obtained from 331 patients. Among them, 211 patients were on prolonged DAPT. Of the patients, 135 (64.0\%) had HPR and 76 (36.0\%) did not have HPR at 1 year. The mean total follow-up period was

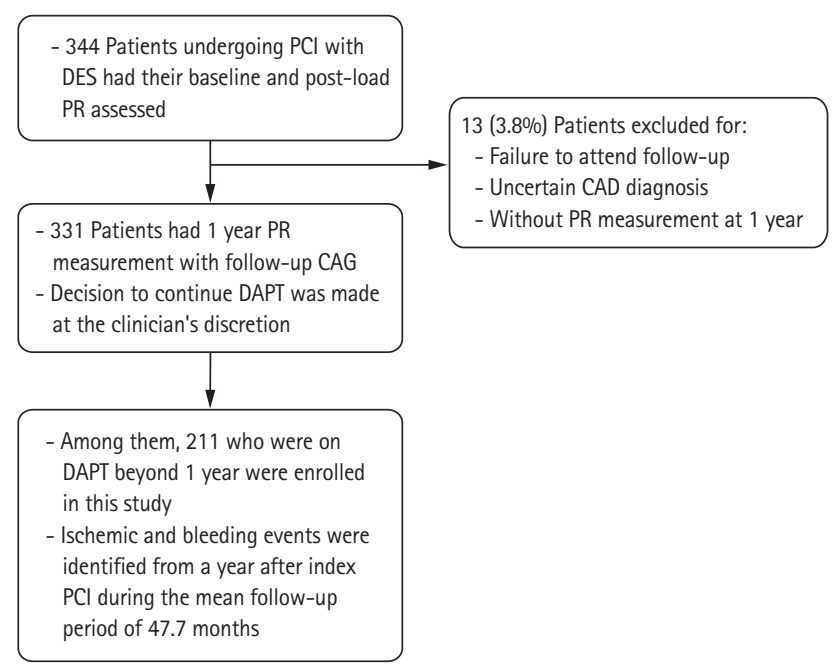

Figure 1. Study flow. PCI, percutaneous coronary intervention; DES, drug-eluting stent; PR, platelet reactivity; CAG, coronary angiography; DAPT, dual antiplatelet therapy; $\mathrm{CAD}$, coronary artery disease.

$47.7 \pm 16.8$ months (Fig. 1).

\section{Baseline characteristics}

The baseline characteristics of the study patients are shown in Table 1. The population had a mean age of $61.9 \pm 10.1$ years and body mass index of $24.8 \pm 3.2 \mathrm{~kg} / \mathrm{m}^{2}$, and $157(74.4 \%)$ were men. The average clopidogrel use duration was $39.3 \pm 24.2$ months. With respect to clinical presentations, 79 (37.4\%) patients were admitted for stable angina, 37 (17.5\%) had unstable angina, 39 (18.4\%) had non-ST-elevation MI, and 56 (26.5\%) had ST-elevation MI. The HPR at 1 year group showed more advanced age $(63.1 \pm 9.9$ years vs. $58.9 \pm 10.0$ years, $p=0.02)$ and higher rates of HTN (83 [61.5\%] vs. 32 [42.1\%], $p=0.007)$ and prior history of TIA/stroke ( $15[11.1 \%]$ vs. $2[2.6 \%], p=0.03)$ than the non-HPR group. Conversely, the non-HPR group showed a higher proportion of male patients (66 [86.8\%] vs. 91 [67.4\%], $p=0.002)$ and current smokers (37 [48.7\%] vs. 46 [34.1\%], $p=0.037$ ) than the HPR group (Table 1 ). Notably, the clinical and angiographic characteristics with medication use were well balanced between the two groups (Table 2).

\section{Relationship between HPR at 1 year and clinical outcomes beyond 1 year}

Survival analysis using the Kaplan-Meier survival anal- 
Table 1. Baseline characteristics

\begin{tabular}{|c|c|c|c|}
\hline \multirow{2}{*}{ Characteristic } & \multicolumn{2}{|c|}{ Group } & \multirow{2}{*}{$p$ value } \\
\hline & $\operatorname{HPR}(\mathrm{n}=135)$ & Non-HPR $(n=76)$ & \\
\hline Age, yr & $63.1 \pm 9.9$ & $58.9 \pm 10.0$ & 0.020 \\
\hline Male sex & $91(67.4)$ & $66(86.8)$ & 0.002 \\
\hline $\mathrm{BMI}, \mathrm{kg} / \mathrm{m}^{2}$ & $24.8 \pm 3.4$ & $24.6 \pm 2.7$ & 0.629 \\
\hline Prior myocardial infarction & $4(3.0)$ & $4(5 \cdot 3)$ & 0.463 \\
\hline Total follow-up period, mo & $46.2 \pm 17 \cdot 3$ & $50.2 \pm 15 \cdot 5$ & 0.096 \\
\hline Smoking & $46(34 \cdot 1)$ & $37(48.7)$ & 0.037 \\
\hline Hypertension & $83(61.5)$ & $32(42.1)$ & 0.007 \\
\hline Diabetes mellitus & $47(34.8)$ & $20(26.3)$ & 0.203 \\
\hline Dyslipidemia & $27(20.0)$ & $17(22.4)$ & 0.684 \\
\hline Atrial fibrillation & $1(0.7)$ & $1(1.3)$ & 1.000 \\
\hline Prior stroke/TIA & $15(11.1)$ & $2(2.6)$ & 0.030 \\
\hline Chronic kidney disease & $8(5 \cdot 9)$ & $1(1.3)$ & 0.161 \\
\hline Hematocrit, \% & $40.1 \pm 5.4$ & $40.8 \pm 6.2$ & 0.433 \\
\hline Blood urea nitrogen, mg/dL & $16.5 \pm 12.6$ & $16.1 \pm 10.8$ & 0.830 \\
\hline Creatinine, $\mu \mathrm{mol} / \mathrm{L}$ & $1.12 \pm 1.12$ & $1.30 \pm 1.78$ & 0.350 \\
\hline Platelet, $10^{3} / \mathrm{L}$ & $260 \pm 66$ & $263 \pm 86$ & 0.754 \\
\hline
\end{tabular}

Values are presented as mean $\pm \mathrm{SD}$ or number (\%).

HPR, high on-clopidogrel platelet reactivity; BMI, body mass index; TIA, transient ischemic attack.

ysis and Cox proportional hazard regression analysis showed that ischemic endpoint events, including cardiovascular death, MI, and stroke/TIA, were significantly increased in patients with than without HPR at 1 year (HR, 2.68; 95\% CI, 1.06 to 6.77; $p=0.036$ ). Meanwhile, any-BARC bleeding events were found to be significantly lower in patients with than without HPR at 1 year (HR, 0.11; 95\% CI, 0.02 to $0.65 ; p=0.015$ ) (Table 3, Fig. 2).

\section{Longitudinal change in PR between index $\mathrm{PCl}$ and 1 year thereafter}

A total of 57 patients with available baseline and postload PRU data were analyzed using repeated measures ANOVA to evaluate the longitudinal change in PR. The PRU level at baseline, post-load, and 1 year after index PCI in the non-HPR group versus the HPR in 1 year group were as follows: $269.8 \pm 61.4$ vs. $290.8 \pm 62.2, p=$ $0.227 ; 194.4 \pm 72.5$ vs. $257.8 \pm 85.6, p=0.007$; and $151.1 \pm$ 36.2 vs. $279.1 \pm 45.9, p<0.001$, respectively. Overall, there was a significant decrease in PR from baseline to 1 year after index PCI in the non-HPR group. However, the HPR group showed high PR from baseline through 1 year. Hence, there was a significant difference in the longitudinal change of PR according to time (within-subject time interaction, $p_{\text {ws-time }}<0.001$ ) and group (between-subject group interaction, $p_{\text {bs-group }}<0.001$ ). Accordingly, there was an interaction by time and group $\left(p_{\text {time }}{ }^{\text {group }}<0.001\right)$ (Table 4, Fig. 3).

In the subgroup analysis of patients with stable angina and ACS, the pattern of longitudinal change differed according to clinical presentation. In patients with stable angina, post-load PR showed a significant decrease compared with the baseline value, which was maintained up to 1 year in the non-HPR group. In contrast, the HPR group showed little change in PR throughout the baseline, post-load, and 1-year periods (Fig. 4A). However, in patients with ACS, the PR of the two groups converged at the post-load period and significantly diverged after 1 year (between-subject group at each time point, $p_{\text {bs-group }}$ each time point $=0.349,0.401$, and $<0.001$ at baseline, postload, and 1 year, respectively). Therefore, there was also

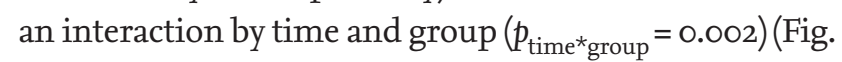
4B). 
Table 2. Baseline characteristics of clinical and angiographic diagnosis and medication use

\begin{tabular}{|c|c|c|c|}
\hline \multirow{2}{*}{ Characteristic } & \multicolumn{2}{|c|}{ Group } & \multirow{2}{*}{$p$ value } \\
\hline & $\operatorname{HPR}(\mathrm{n}=135)$ & Non-HPR $(n=76)$ & \\
\hline \multicolumn{4}{|l|}{ Clinical diagnosis } \\
\hline Stable angina & $50(37.0)$ & $29(38.2)$ & 0.872 \\
\hline Unstable angina & $23(17.0)$ & $14(18.4)$ & 0.800 \\
\hline Non-STEMI & $22(16.3)$ & $17(22.4)$ & 0.275 \\
\hline STEMI & $40(29.6)$ & $16(21.1)$ & 0.197 \\
\hline \multicolumn{4}{|l|}{ Angiographic diagnosis } \\
\hline 1 Vessel disease & $57(42.2)$ & $37(48.7)$ & 0.365 \\
\hline 2 Vessel disease & $39(28.9)$ & $23(30.3)$ & 0.833 \\
\hline 3 Vessel disease & $39(28.9)$ & $16(21.1)$ & 0.213 \\
\hline \multicolumn{4}{|l|}{ Medication } \\
\hline ACEi/ARB & $104(77.0)$ & $55(72.4)$ & 0.450 \\
\hline Beta blocker & $96(71.1)$ & $46(60.5)$ & 0.116 \\
\hline Calcium channel blocker & $66(48.9)$ & $29(38.2)$ & 0.133 \\
\hline Nitrate & $3(2.2)$ & $4(5 \cdot 3)$ & 0.255 \\
\hline Statin & $124(91.9)$ & $71(93 \cdot 4)$ & 0.679 \\
\hline Proton pump inhibitor & $6(4 \cdot 4)$ & $6(7.9)$ & 0.357 \\
\hline
\end{tabular}

Values are presented as number (\%).

HPR, high on-clopidogrel platelet reactivity; STEMI, ST segment elevation myocardial infarction; ACEi, angiotensin converting enzyme inhibitor; ARB, angiotensin receptor blocker.

Table 3. Relationship between HPR at 1 year and clinical outcomes beyond 1 year

\begin{tabular}{|c|c|c|c|c|c|}
\hline Variable & $\operatorname{HPR}(n=135)$ & Non-HPR $(n=76)$ & Log-rank $p$ value ${ }^{b}$ & $\mathrm{HR}(95 \% \mathrm{CI})$ & $p$ value $^{c}$ \\
\hline Ischemic endpoint $^{\mathrm{a}}$ & $20.0(27)$ & $7 \cdot 9(6)$ & 0.016 & $2.68(1.06-6.77)$ & 0.036 \\
\hline Any-BARC bleeding & $2.2(3)$ & $13.2(10)$ & 0.046 & $0.11(0.02-0.65)$ & 0.015 \\
\hline
\end{tabular}

Values are presented as percentage (number).

HPR, high on-clopidogrel platelet reactivity; HR, hazard ratio; CI, confidence interval; BARC, Bleeding Academic Research Consortium.

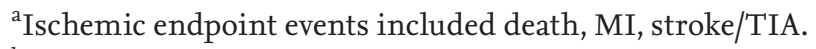

${ }^{\mathrm{b}}$ Log-rank test was used to compare survival outcomes between the two groups.

${ }^{\mathrm{c}}$ Multivariable-adjusted Cox proportional regression hazard analysis was performed using covariates including age $>65$ years, sex, prior myocardial infarction, stroke/transient ischemic attack, hypertension, diabetes mellitus, atrial fibrillation, dyslipidemia, chronic kidney disease, and smoking status.

\section{DISCUSSION}

In this study, we sought to evaluate the clinical implications of HPR at 1 year on future cardiovascular and bleeding outcomes beyond 1 year as well as the longitudinal change of $\mathrm{PR}$ in patients with prolonged clopidogrel therapy after DES-based PCI. There were several key findings from this study: (1) the HPR at 1 year group showed a significant increase in ischemic cardiovascular outcomes at the expense of increased bleeding; (2) the pattern of longitudinal change was significantly different between the HPR and non-HPR groups at 1 year; and (3) there were different patterns of longitudinal change according to the clinical presentation. In patients with ACS, the PR of the two groups converged at post-load and significantly widened at 1 year after index PCI. 
There have been previous reports on the impact of HPR on clinical outcomes, mostly focusing on ischemic events. It is well known that post-load on-clopidogrel reactivity < 208 PRU is associated with a lower risk of cardiovascular events, and an increasing num-

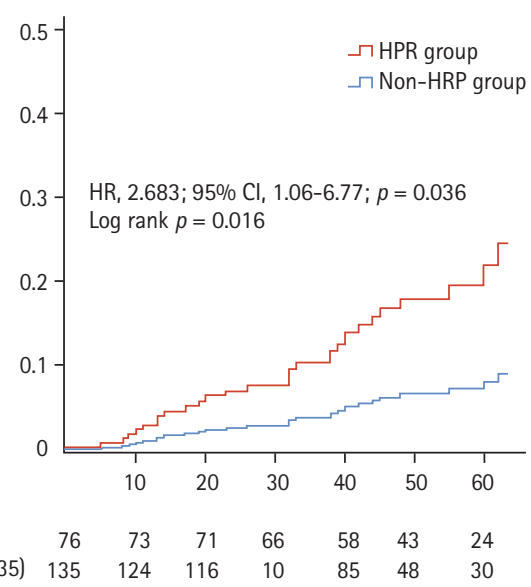

Figure 2. Ischemic outcomes. The overall ischemic event rate during the follow-up period is shown. A cumulative hazard function curve shows a significant increase in the ischemic events in patients with high on-treatment platelet reactivity (HPR). HR, hazard ratio; CI, confidence interval. ber of studies have found that patients with HPR have a higher risk of cardiovascular death, non-fatal MI, and stent thrombosis $[14,16]$. The platelet reactivity and clinical outcomes after coronary artery implantation of drug-eluting stents (ADAPT-DES) study found the counterbalancing effects of hemorrhagic and ischemic complications, in that HPR was strongly associated with stent thrombosis and MI and was inversely related to bleeding [7]. In this study, we attempted to investigate whether on-clopidogrel PR at 1 year could predict future cardiovascular outcomes in patients on prolonged DAPT with aspirin and clopidogrel beyond 1 year. According to our expectations, HPR at 1 year was associated with increased ischemic cardiovascular endpoints, including death, MI, and stroke/TIA, whereas non-HPR at 1 year was associated with a higher incidence of bleeding, which was in line with the results of previous studies that investigated clinical outcomes within 1 year [17]. Therefore, we re-confirmed the counterbalancing effect of hemorrhagic and ischemic complications, and determined that a tailored strategy is needed in patients with HPR on prolonged DAPT therapy.

Another interesting finding was platelet variability. Several previous studies have measured serial PR and investigated the evolving pattern and optimal timing of

Table 4. Longitudinal change in PR between index PCI and 1 year thereafter

\begin{tabular}{|c|c|c|c|c|}
\hline \multirow{2}{*}{ Variable } & \multicolumn{3}{|c|}{ Time } & \multirow{2}{*}{$p$ value ${ }^{a}$} \\
\hline & Baseline & Post-load & At 1-year & \\
\hline \multicolumn{5}{|l|}{ Total $(\mathrm{n}=57)$} \\
\hline Non-HPR group $(\mathrm{n}=20)$ & $269.8 \pm 61.4$ & $194.4 \pm 72.5$ & $154.1 \pm 36.2$ & $p_{\text {ws-time }}<0.001$ \\
\hline HPR group $(\mathrm{n}=37)$ & $290.8 \pm 62.2$ & $257.8 \pm 85.6$ & $279.1 \pm 45 \cdot 9$ & $P_{\text {bs-group }}<0.001$ \\
\hline Pbs-group at each time point & 0.227 & 0.007 & $<0.001$ & $P_{\text {time* }}{ }_{\text {group }}<0.001$ \\
\hline \multicolumn{5}{|l|}{ Stable angina $(\mathrm{n}=19)$} \\
\hline Non-HPR group $(\mathrm{n}=8)$ & $276.9 \pm 64.6$ & $161.8 \pm 69.2$ & $139 \cdot 3 \pm 44 \cdot 7$ & $p_{\mathrm{ws}-\mathrm{time}}=0.001$ \\
\hline $\operatorname{HPR} \operatorname{group}(\mathrm{n}=11)$ & $300.3 \pm 49 \cdot 7$ & $297.6 \pm 62.7$ & $301.9 \pm 46.4$ & $P_{\text {bs-group }}<0.001$ \\
\hline$P_{\mathrm{bs} \text {-group at each time point }}$ & 0.384 & $<0.001$ & $<0.001$ & $p_{\text {time }}{ }_{\text {group }}=0.001$ \\
\hline \multicolumn{5}{|c|}{ Acute coronary syndrome $(n=38)$} \\
\hline Non-HPR group $(\mathrm{n}=12)$ & $265.1 \pm 61.8$ & $216.1 \pm 68.8$ & $163.9 \pm 26.9$ & $p_{\text {ws-time }}<0.001$ \\
\hline $\operatorname{HPR}$ group $(\mathrm{n}=26)$ & $286.8 \pm 67 \cdot 3$ & $240.9 \pm 89.4$ & $269.4 \pm 43.0$ & $P_{\text {bs-group }}=0.006$ \\
\hline Pbs-group at each time point & 0.349 & 0.401 & $<0.001$ & $P_{\text {time }}{ }^{*}$ group $=0.002$ \\
\hline
\end{tabular}

Values are presented as mean $\pm \mathrm{SD}$.

PR, platelet reactivity; PCI, percutaneous coronary intervention; HPR, high on-treatment platelet reactivity; ws-time, within-subject time interaction; bs-group, between-subject group interaction.

${ }^{a}$ Repeated-measures analysis of variance was used to compare temporal change in PR. 
on-clopidogrel PR. Campo et al. [8] previously reported a decrease in PR from baseline to 1 month after the index PCI. In that study, $27 \%$ of the patients showed significant PR variability between the baseline and 1-month values; however, the variability was minimal (0.7\%) from 1 month to 6 months. This result was mainly attributed to the change in baseline poor responders to full responders [8].

In the Testing Platelet Reactivity in Patients Undergoing Elective Stent Placement on Clopidogrel to Guide Alternative Therapy with Prasugrel (TRIGGER-PCI) study, Trenk et al. [18] assessed the temporal variability

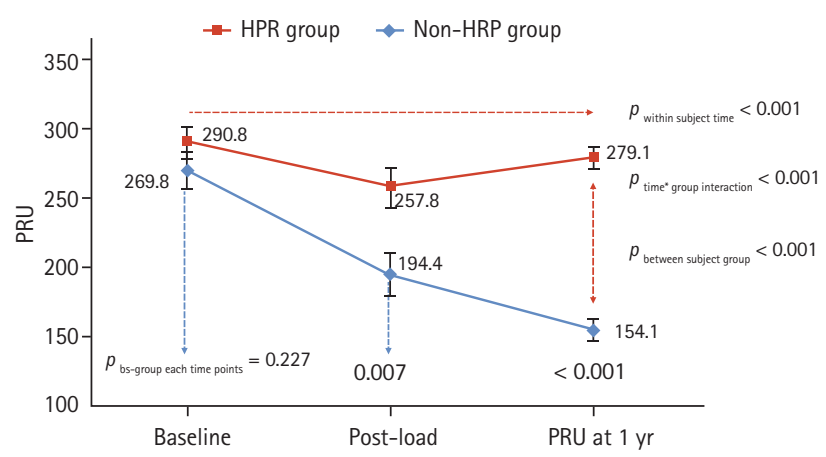

Figure 3. Overall longitudinal change in platelet reactivity (PR) in all patients. The $\mathrm{P}_{2} \mathrm{Y}_{12}$ reaction unit (PRU) level at baseline, post-load, and 1 year after index percutaneous coronary intervention (PCI) were analyzed using repeated measures analysis of variance to evaluate the longitudinal change in PR. PR significantly decreased from post-load to 1 year after index PCI in the non-high on-platelet reactivity (HPR) group. Conversely, the HPR group showed high PR from baseline through 1 year. bs-group, between-subject group interaction. in patients with stable angina who were randomized to receive either prasugrel or clopidogrel, among patients with HPR on-clopidogrel therapy. There was a small decrease in PR from baseline to 3 months, and $29.6 \%$ of the patients reached a PR of $<208$ in the clopidogrel group. However, the prasugrel group showed a substantial decrease in PR at 3 months, and $94.1 \%$ of the patients reached a $P R$ of $<208$. There was no significant change in PR between 3 and 6 months in either group [18]. Our study had a similar finding in the stable angina group, which showed little change in PR between post-load and 1 year, suggesting that the HPR status in these patients at the post-load period may be enough to predict longer-term response to prolonged clopidogrel therapy. However, an interesting finding from our study was that in the HPR group, the PR at post-load was less than that at 1 year, which was more pronounced in patients with ACS, suggesting a possible rebound phenomenon of PR. Until recently, only a few reports have demonstrated this rebound phenomenon of PR in the long-term follow-up period. Tello-Montoliu et al. [19] and Yun et al. [20] similarly observed increased PR in patients with ACS followed up for 6 months. These fluctuations of $\mathrm{PR}$, particularly in patients with ACS, are believed to be affected by multiple factors, including genetic factors, environmental factors, and concomitant medications $[5,8]$. Another possible explanation for this finding is intra-individual variability in PR. The Escalating Clopidogrel by Involving a Genetic Strategy-Thrombolysis In Myocardial Infarction 56 (ELEVATE-TIMI 56) trial demonstrated that $15.7 \%$ of patients taking clopidogrel showed a change in responder status when tested at two
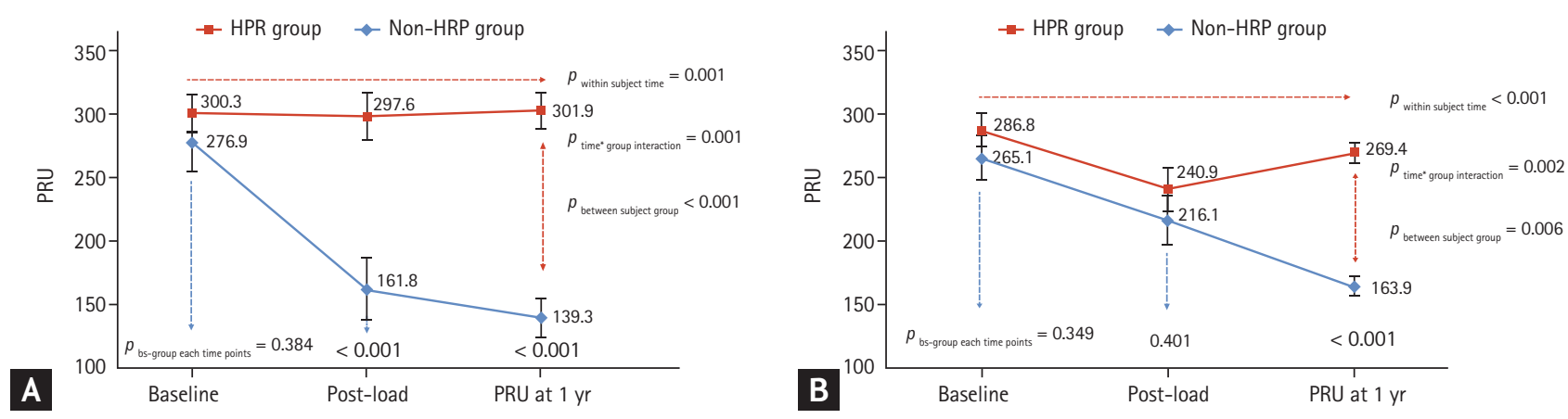

Figure 4. Longitudinal change in platelet reactivity (PR) according to the clinical presentation. (A) The longitudinal change in PR in patients with stable angina. (B) The longitudinal change in PR in patients with acute coronary syndrome. HPR, high on-platelet reactivity; PRU, $\mathrm{P}_{2} Y_{12}$ reaction unit; bs-group, between-subject group interaction. 
different time points [21]. In this regard, testing PR at a single time point or soon after loading time or early in the clinical presentation might not be sufficient to tailor antiplatelet therapy.

There have been studies evaluating the possible role of prolonged therapy with the potent $\mathrm{P}_{2} \mathrm{Y}_{12}$ inhibitor ticagrelor on long-term outcomes in patients with a history of previous PCI. In the Prevention of Cardiovascular Events in Patients With Prior Heart Attack Using Ticagrelor Compared to Placebo on a Background of Aspirin-Thrombolysis in Myocardial Infarction 54 (PEGASUS-TIMI 54) study, patients with previous MI and on aspirin were randomized to receive either ticagrelor 90 or $60 \mathrm{mg}$ twice daily or placebo [22]. In that study, during a median follow-up period of 33 months, ticagrelor significantly reduced the composite primary efficacy endpoints (cardiovascular death, MI, or stroke) as compared with placebo (7.85\%, 7.77\%, and 9.04\%; $p=0.008)$, although there was increased bleeding. The most recent ticagrelor in patients with diabetes and stable CAD with a history of previous percutaneous coronary intervention (THEMIS-PCI) trial randomized patients with diabetes and a history of previous PCI to receive either ticagrelor or placebo, to determine whether there is a benefit in terms of long-term cardiovascular outcomes. The results showed fewer primary efficacy outcomes in patients treated with ticagrelor than in those who received placebo $(7.3 \%$ vs. 8.6\%; HR, 0.85 ; $95 \% \mathrm{CI}, 0.74$ to 0.97 ; $p=0.013$ ) over a median follow-up of 33 months. Again, there was an increase in Thrombolysis in Myocardial Infarction major bleeding in the ticagrelor group than in the placebo group (2.0\% vs. $1.1 \%$; HR, 2.03 ; $95 \%$ CI, 1.48 to 2.76 ; $p<0.0001$ ) [23]. Although these studies did not compare the impact of ticagrelor with that of clopidogrel, the investigators clearly showed the benefit of long-term platelet inhibition with a potent $\mathrm{P}_{2} \mathrm{Y}_{12}$ inhibitor on future cardiovascular outcomes. Taking the results together, it might be justifiable for selected patients with on-clopidogrel HPR to switch to a potent $\mathrm{P}_{2} \mathrm{Y}_{12}$ inhibitor even beyond 1 year, especially in those with high ischemic risks, such as postMI patients or those with diabetes. Given the variability of PR in patients treated with clopidogrel, particularly in those with ACS, it might be reasonable to measure PR at 1 year to define the HPR status for predicting future outcomes as well as for tailoring antiplatelet therapy in the long-term period.
This study had several limitations. First, owing to its retrospective design and relatively small sample size, selection bias may be present. Nevertheless, our study provided real-world findings that deserve further investigation in future studies with a randomized design, a larger population, and a longer follow-up period. Second, we only measured and analyzed 1-year PR along with the baseline and post-load values. PR measured at more time points may provide a better understanding of PR variability. Third, the differences in baseline characteristics, such as smoking status, may have acted as unknown confounders affecting PR. Further randomized studies with balanced baseline characteristics of study subjects might overcome these limitations [24,25].

In conclusion, HPR at 1 year may be useful as a surrogate marker for predicting ischemic and bleeding events beyond 1 year in patients on prolonged DAPT. In patients with ACS, post-load PR may not reliably reflect on-clopidogrel $\mathrm{PR}$. These findings suggest that PR monitoring at 1-year post-PCI may help clinicians optimize antiplatelet management in the long-term follow-up period.

\section{KEY MESSAGE}

1. High on-treatment platelet reactivity at 1 year may be useful as a surrogate marker for predicting ischemic and bleeding events beyond 1 year in patients on prolonged dual antiplatelet therapy.

2. In patients with acute coronary syndrome, post-load platelet reactivity (PR) may not reliably reflect on-clopidogrel $\mathrm{PR}$.

3. PR monitoring at 1-year post-percutaneous coronary intervention may help clinicians optimize antiplatelet management in the longterm follow-up period.

\section{Conflict of interest}

No potential conflict of interest relevant to this article was reported.

\section{Acknowledgments}

The authors thank the cath lab and clinical staff involved in this study. 


\section{REFERENCES}

1. Kitamura T, Obara H, Takashima Y, et al. World Health Assembly agendas and trends of international health issues for the last 43 years: analysis of World Health Assembly agendas between 1970 and 2012. Health Policy 2013;110:198-206.

2. Holmes DR Jr, Kereiakes DJ, Garg S, et al. Stent thrombosis. J Am Coll Cardiol 2010;56:1357-1365.

3. Mauri L, Hsieh WH, Massaro JM, Ho KK, D'Agostino R, Cutlip DE. Stent thrombosis in randomized clinical trials of drug-eluting stents. N Engl J Med 2007;356:1020-1029.

4. Valgimigli M, Bueno H, Byrne RA, et al. 2017 ESC focused update on dual antiplatelet therapy in coronary artery disease developed in collaboration with EACTS: the task force for dual antiplatelet therapy in coronary artery disease of the European Society of Cardiology (ESC) and of the European Association for Cardio-Thoracic Surgery (EACTS). Eur Heart J 2018;39:213-26o.

5. Mega JL, Simon T. Pharmacology of antithrombotic drugs: an assessment of oral antiplatelet and anticoagulant treatments. Lancet 2015;386:281-291.

6. Wiviott SD, Steg PG. Clinical evidence for oral antiplatelet therapy in acute coronary syndromes. Lancet 2015;386:292-302.

7. Stone GW, Witzenbichler B, Weisz G, et al. Platelet reactivity and clinical outcomes after coronary artery implantation of drug-eluting stents (ADAPT-DES): a prospective multicentre registry study. Lancet 2013;382:614-623.

8. Campo G, Parrinello G, Ferraresi P, et al. Prospective evaluation of on-clopidogrel platelet reactivity over time in patients treated with percutaneous coronary intervention relationship with gene polymorphisms and clinical outcome. J Am Coll Cardiol 2011;57:2474-2483.

9. Alexopoulos D, Xanthopoulou I, Siapika A, et al. Evolving pattern of on-prasugrel and on-ticagrelor platelet reactivity over time in ST elevation myocardial infarction patients. Int J Cardiol 2013;168:629-630.

10. Mehran R, Rao SV, Bhatt DL, et al. Standardized bleeding definitions for cardiovascular clinical trials: a consensus report from the Bleeding Academic Research Consortium. Circulation 2011;123:2736-2747.

11. Ndrepepa G, Schuster T, Hadamitzky M, et al. Validation of the Bleeding Academic Research Consortium definition of bleeding in patients with coronary artery disease undergoing percutaneous coronary intervention. Circu- lation 2012;125:1424-1431.

12. Malinin A, Pokov A, Spergling M, et al. Monitoring platelet inhibition after clopidogrel with the VerifyNow-P2Y12(R) rapid analyzer: the VERIfy Thrombosis risk Assessment (VERITAS) study. Thromb Res 2007;119:277-284.

13. Tantry US, Bonello L, Aradi D, et al. Consensus and update on the definition of on-treatment platelet reactivity to adenosine diphosphate associated with ischemia and bleeding. J Am Coll Cardiol 2013;62:2261-2273.

14. Price MJ, Angiolillo DJ, Teirstein PS, et al. Platelet reactivity and cardiovascular outcomes after percutaneous coronary intervention: a time-dependent analysis of the Gauging Responsiveness with a VerifyNow P2Y12 assay: Impact on Thrombosis and Safety (GRAVITAS) trial. Circulation 2011;124:1132-1137.

15. Breet NJ, van Werkum JW, Bouman HJ, et al. Comparison of platelet function tests in predicting clinical outcome in patients undergoing coronary stent implantation. JAMA 2010;303:754-62.

16. Aradi D, Komocsi A, Vorobcsuk A, et al. Prognostic significance of high on-clopidogrel platelet reactivity after percutaneous coronary intervention: systematic review and meta-analysis. Am Heart J 2010;160:543-551.

17. Mauri L, Kereiakes DJ, Yeh RW, et al. Twelve or 30 months of dual antiplatelet therapy after drug-eluting stents. N Engl J Med 2014;371:2155-2166.

18. Trenk D, Stone GW, Gawaz M, et al. A randomized trial of prasugrel versus clopidogrel in patients with high platelet reactivity on clopidogrel after elective percutaneous coronary intervention with implantation of drug-eluting stents: results of the TRIGGER-PCI (Testing Platelet Reactivity In Patients Undergoing Elective Stent Placement on Clopidogrel to Guide Alternative Therapy With Prasugrel) study. J Am Coll Cardiol 2012;59:2159-2164.

19. Tello-Montoliu A, Rivera J, Hernandez D, et al. Temporal changes in platelet response in acute coronary syndrome patients with prasugrel and clopidogrel after stent implantation. Circ J 2018;82:353-360.

20. Yun KH, Cho JY, Rhee SJ, Oh SK. Temporal variability of platelet reactivity in patients treated with clopidogrel or ticagrelor. Korean Circ J 2019;49:1052-1061.

21. Hochholzer W, Ruff CT, Mesa RA, et al. Variability of individual platelet reactivity over time in patients treated with clopidogrel: insights from the ELEVATE-TIMI 56 trial. J Am Coll Cardiol 2014;64:361-368. 
22. Bonaca MP, Bhatt DL, Cohen M, et al. Long-term use of ticagrelor in patients with prior myocardial infarction. $\mathrm{N}$ Engl J Med 2015;372:1791-1800.

23. Bhatt DL, Steg PG, Mehta SR, et al. Ticagrelor in patients with diabetes and stable coronary artery disease with a history of previous percutaneous coronary intervention (THEMIS-PCI): a phase 3, placebo-controlled, randomised trial. Lancet 2019;394:1169-1180.

24. Barbash GI, Reiner J, White HD, et al. Evaluation of paradoxic beneficial effects of smoking in patients receiving thrombolytic therapy for acute myocardial infarction: mechanism of the "smoker's paradox" from the GUSTO-I trial, with angiographic insights. Global Utilization of Streptokinase and Tissue-Plasminogen Activator for Occluded Coronary Arteries. J Am Coll Cardiol 1995;26:12221229.

25. Crimi G, Somaschini A, Cattaneo M, et al. Cigarette smoking reduces platelet reactivity independently of clopidogrel treatment in patients with non-ST elevation acute coronary syndromes. Platelets 2018;29:309-311. 